\title{
Transbronchial cryobiopsy: how to do it safely?
}

\author{
Jasleen Pannu ${ }^{1}$, Van Holden ${ }^{2}$, Christian Ghattas ${ }^{1}$ \\ ${ }^{1}$ Division of Pulmonary and Critical Care Medicine, Ohio State University Medical Center, Columbus, OH, USA; ${ }^{2}$ Division of Pulmonary and \\ Critical Care Medicine, University of Maryland, Baltimore, MD, USA \\ Contributions: (I) Conception and design: J Pannu, C Ghattas; (II) Administrative support: None; (III) Provision of study materials or patients: None; \\ (IV) Collection and assembly of data: All authors; (V) Data analysis and interpretation: All authors; (VI) Manuscript writing: All authors; (VII) Final \\ approval of manuscript: All authors. \\ Correspondence to: Jasleen Pannu, MBBS. Division of Pulmonary and Critical Care Medicine, Ohio State University Medical Center, 410 W 10 th Ave, \\ Columbus, OH, USA. Email: Jasleen.Pannu@osumc.edu.
}

\begin{abstract}
Transbronchial cryobiopsy (TBC) is a bronchoscopic procedure for obtaining larger and higher quality peripheral lung biopsies with less crush artifact as compared to conventional transbronchial lung biopsy. It has demonstrated a higher diagnostic yield than the traditional forceps biopsy and thus has generated significant interest as a minimally invasive diagnostic tool for the diagnosis of diffuse parenchymal lung disease (DPLD) in recent years. However, the possibility of intra-operative bleeding, pneumothorax, and other postoperative complications have raised significant concerns. More recently, studies on postoperative complications, morbidity, and mortality as well as the use of techniques to prevent complications of this procedure have been published. Expert guidelines with graded recommendations based on the GRADE approach have been released. Ours is an updated review of practices to perform TBC safely; classified as pre-procedural, intraoperative and postoperative practices that can be adopted and applied in clinical practice based on current evidence to decrease the incidence of adverse outcomes.
\end{abstract}

Keywords: Transbronchial cryobiopsy (TBC); diffuse parenchymal lung disease (DPLD)

Received: 26 December 2019; Accepted: 09 March 2020; Published: 10 April 2021.

doi: $10.21037 /$ shc.2020.03.05

View this article at: http://dx.doi.org/10.21037/shc.2020.03.05

\section{Introduction}

Transbronchial cryobiopsy (TBC) is a bronchoscopic procedure for obtaining larger and higher quality peripheral lung biopsies with less crush artifact as compared to conventional transbronchial lung biopsy $(1,2)$. It has demonstrated a higher diagnostic yield than the traditional forceps biopsy and thus has generated significant interest as a minimally invasive diagnostic tool for the diagnosis of diffuse parenchymal lung disease (DPLD) in recent years (3-5). However, the possibility of intra-operative bleeding, pneumothorax and other postoperative complications have raised significant concerns. An expert statement from the cryobiopsy working group on the safety and utility of TBC in the diagnosis of DPLD was released, and a call for standardization of the procedure was made in January 2018 (4). Grading of the level of evidence per GRADE
(Grading of Recommendations Assessment, Development, and Evaluation) criteria could not be made in this document due to the low quality of evidence available; however, suggestions on safe procedural practices were outlined and standardization of practices was emphasized.

Since then, more studies on postoperative complications, morbidity, and mortality as well as the use of additional techniques to prevent complications of this procedure have been published (6-9). More recently, expert guidelines with graded recommendations based on the GRADE approach have been released (10). Ours is an updated review of practices to perform TBC safely; classified as pre-procedural, intraoperative and postoperative practices that can be adopted and applied in clinical practice based on current evidence to decrease the incidence of adverse outcomes. 


\section{How to perform TBC safely: pre-procedural considerations}

A detailed and cautious pre-operative assessment can reduce complications related to TBC. This procedure is associated with a higher risk of procedural morbidity and mortality as compared to the conventional transbronchial forceps biopsy $(6,7,11)$. Eligibility and indications for TBC should be reviewed for each patient, and the complication profile discussed in detail with the patient well ahead of the procedure $(2,5)$. Once the appropriate indication for the procedure is confirmed, individual patient characteristics and any contraindications should be reviewed to determine the safety of performing this procedure followed by proper optimization of these patients $(4,6-8,12)$.

\section{Patient eligibility: indications to perform transbronchial cryobiopsy}

Transbronchial cryobiopsy is commonly indicated for DPLD to provide tissue analysis following multidisciplinary discussion (MDD), especially when radiological and clinical workup does not yield a definitive diagnosis $(4,8,13)$. Besides this, the use of this procedure for other indications such as biopsy of lung nodules, lung transplant surveillance, suspected small airway disease, and presence of pulmonary infiltrates in immunocompromised patients is also reported (4,13-17).

Idiopathic pulmonary fibrosis (IPF), sarcoidosis, connective tissue diseases, drugs, environmental inhalants, and hypersensitivity pneumonitis (HP) are the most common causes of DPLD (18). Since invasive diagnostic approaches such as TBC or surgical lung biopsy (SLB) are associated with potential risks and complications (4), proper patient selection is crucial. Patients should undergo a detailed pre-procedural workup, including a complete history and clinical examination, biochemical and serological testing, pulmonary function testing, and a high-resolution computerized tomography scan (HRCT) of the chest (4). A multidisciplinary discussion (MDD) for diagnosing DPLD is now considered gold standard $(8,13,19)$. This multi-disciplinary committee consisting of a team of experienced pulmonologists, pathologists, and radiologists reviews each case and analyzes the clinical presentation, laboratory, and radiological testing, to arrive at a diagnosis and to determine the additional need for a lung biopsy (13).

\section{Patient eligibility, contraindications, and pre-procedural optimization}

Once the appropriate indication is confirmed, the presence of any contraindications to performing TBC should be ruled out. Patient preference and presence of local expertise in TBC or SLB should also be evaluated (10). The precautions outlined below can be considered to optimize the patient for a safe TBC procedure.

\section{Bleeding diathesis and anticoagulation}

Thrombocytopenia (platelets less than 50,000 per cubic millimeter, $\mathrm{mm}^{3}$ ) is considered a contraindication to TBC and transbronchial biopsy $(4,20)$. Also, patients using clopidogrel or other newer antiplatelet agents should not undergo TBC unless all potential risks of bleeding are corrected before the procedure (4). The use of antiplatelet agents like clopidogrel is strongly associated with an increased risk of bleeding with transbronchial biopsy. Ernst et al. conducted a prospective study of 604 patients who underwent transbronchial lung biopsy (20). Sixteen of the 18 patients $(89 \%)$ who were on clopidogrel within 24 hours of the bronchoscopy, experienced bleeding as compared to 20 out of 574 control patients (3.4\%). The study had to be stopped early due to this significant difference between groups. Thirty-four percent of the bleeding episodes were moderate and $27 \%$ were considered significant bleeds.

Additionally, concomitant use of both clopidogrel and aspirin was associated with bleeding in $100 \%$ of patients (50\% moderate bleeding and 50\% major bleeding) (20). In another retrospective case series of 25 patients with platelet count $<60,000 / \mathrm{mm}^{3}$ who underwent transbronchial biopsies, one patient had a fatal bleeding outcome $(20,21)$. However, in a large prospective trial that included 1,217 patients, lowdose aspirin did not show an increase in the risk of bleeding following transbronchial lung biopsy (TBLB) (22).

Recommendations regarding stopping antiplatelet agents are similar to those for performing TBLB $(20,23,24)$. Based on the study results mentioned above and supported by the current British Thoracic Society (BTS) guidelines, the recommendation is to hold clopidogrel and newer antiplatelet agents 5-7 days before transbronchial biopsies (24). It is still reasonable, if feasible, to hold the aspirin for five days before the procedure; however, it is not considered an absolute contraindication (25). 
There are no comparative studies to determine the risk of bleeding from TBLB when the patients are receiving oral anticoagulants; however, the BTS guidelines recommend discontinuation of warfarin 5 days before the endoscopic procedure for low-risk patients and that INR (International Normalized Ratio) should be checked before the procedure to ensure its level is less than 1.5. In patients at high risk of thrombo-embolic events, warfarin should be discontinued 5 days prior and low molecular weight heparin (LMWH) can be started two days after discontinuation. LMWH should be omitted on the day of the procedure and it can be resumed the evening of the procedure day with the usual daily dose (24).

Uremia also alters platelet function, and it is better to undergo bronchoscopy shortly after hemodialysis. There is some evidence to suggest that desmopressin improves platelet function in uremic patients, and it can be administered before the scheduled bronchoscopy (26).

Underlying liver insufficiency is often associated with coagulation abnormalities; consider coagulation studies before the planned procedure and correct the anomalies in patients with suspected liver disease (27).

\section{Pulmonary function}

Impaired pulmonary function is associated with a higher risk of complications based on data reported from studies on surgical lung biopsy (SLB) (4). Several studies have used cut off limits on the pulmonary function to minimize the risk of post-procedural complications recommending that diffusion capacity of the lungs for carbon monoxide (DLCO) less than $35 \%$ of predicted or forced vital capacity (FVC) less than $50 \%$ of predicted could be considered as a relative contraindication to TBC $(1,4,8,28)$. Other exclusion criteria for some TBC studies were forced expiratory volume in the first second (FEV1) of less than 0.8 liters (L) or less than $50 \%$ of predicted, FVC less than $50 \%$ of predicted, and DLCO less than $35 \%$ or $50 \%$ of predicted $(1,8,28)$. Not all prior studies used a cut off for the level of hypoxemia as an exclusion criterion for TBC. The partial pressure of oxygen $(\mathrm{PaO} 2)$ of less than 55-60 millimeters of mercury $(\mathrm{mmHg})$ on room air or supplemental oxygen requirement of more than $2 \mathrm{~L}$ per minute is considered a contraindication in some studies $(8,29,30)$. Patients with lower FVC or DLCO, higher radiological fibrotic score, or usual interstitial pneumonia (UIP) pattern found on histology were also at higher risk of post-TBC complications $(2,8,10)$.

\section{Acute deterioration}

Inpatients and patients experiencing an acute respiratory decline over the last 30 days (increased dyspnea, increased need for supplemental oxygen and growing pulmonary infiltrates) are at an increased risk of death within 30 days post-procedure and should not undergo TBC. In a recent study of 197 patients who underwent TBC, $25 \% 30$-day mortality was reported for inpatients ( 2 of 8 total inpatients) compared to $1.1 \%$ for outpatients ( 2 of 189 total outpatients) (6). Another retrospective study of 159 patients who underwent TBC showed that the hospitalized group of patients also experienced a higher rate of pneumothorax, persistent air leak, transfer to the ICU, and mortality at 30 days when compared to the outpatient group (7).

\section{Obesity}

Obesity is considered a relative contraindication. In one retrospective study of 69 cases, 3 patients with BMI $>30 \mathrm{Kg} / \mathrm{m}^{2}$ could not undergo TBC for desaturating during the procedure; however, all cases were performed through rigid bronchoscopy and type of ventilation was not reported (28).

\section{Pulmonary hypertension}

Pulmonary hypertension is a relative contraindication to TBC in the absence of sufficient data (25). Patients with clinical or radiological signs of pulmonary hypertension should undergo a preoperative evaluation of their pulmonary artery pressure by echocardiography or right heart catheterization. An estimated systolic pulmonary artery pressure $>50 \mathrm{mmHg}$ on echocardiography is indicative of a high risk of pulmonary hypertension and operative complications $(31,32)$.

Key points to consider before performing TBC are summarized in Table 1.

\section{How to perform TBC safely: intraprocedural considerations}

Safety practices are essential to minimize the risk of complications when performing TBC. A step by step procedural process as well as an evidence-based review is discussed below.

\section{Anesthesia plan}

All patients undergoing TBC should be deeply sedated 
Table 1 Key points: pre-procedural safety checklist

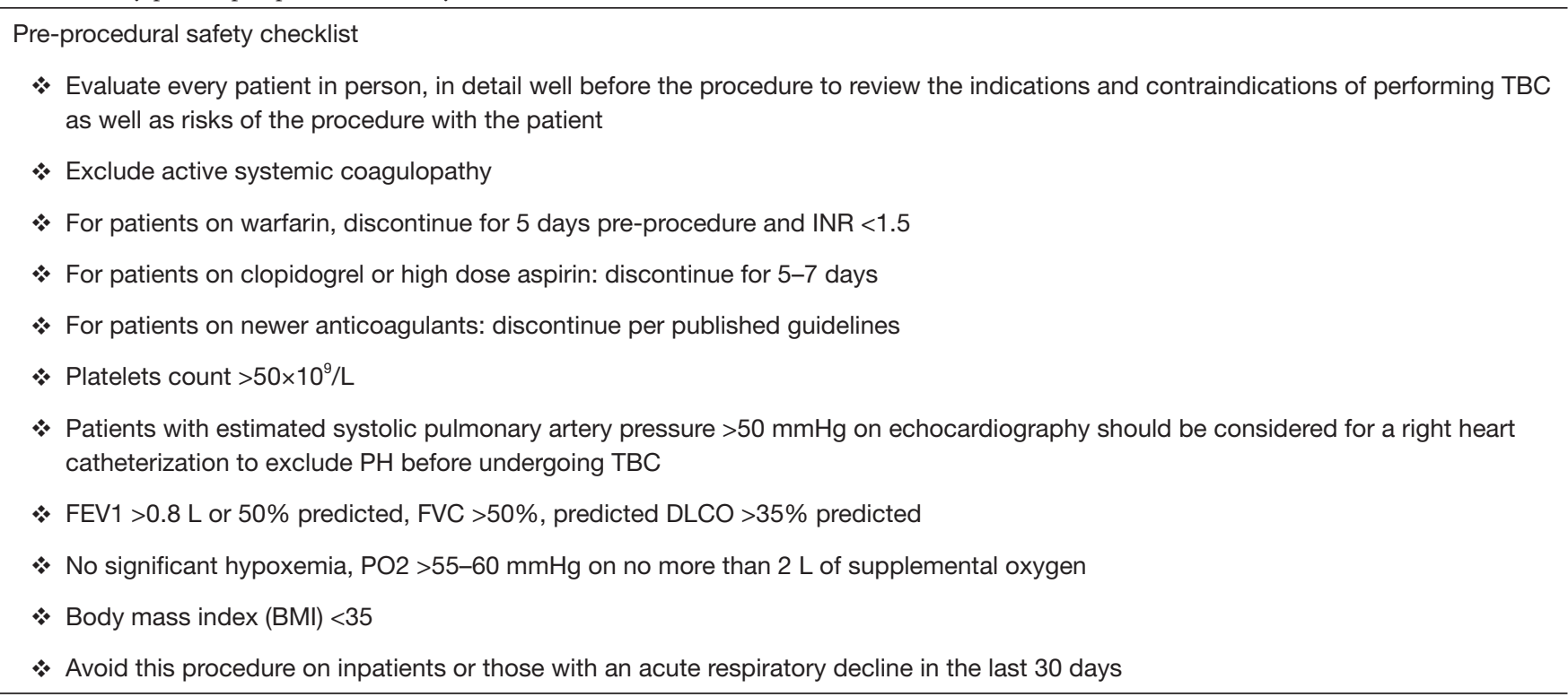

or under general anesthesia in order to best suppress coughing and movement during the procedure, which would otherwise increase the risk of pneumothorax or probe displacement and airway injury $(2,4,33)$. Keeping the patient spontaneously breathing by avoiding neuromuscular paralysis as well as keeping the endotracheal tube cuff deflated-hence decreasing positive pressure ventilation can reduce the risk of pneumothorax during procedure (2). Jet ventilation has been used safely and successfully with the use of rigid bronchoscopy for TBC $(2,4,8,34)$. However, it would require patients to be under neuromuscular paralysis.

\section{Staff preparation}

TBC involves coordination between multiple personnel during the procedure to ensure its appropriate execution. The process should be reviewed step by step with the team and roles assigned beforehand to improve communication. Closed-loop communication should be practiced during the procedure (35). Similar to the proceduralist, trained staff to perform this procedure is favorable. Simulation-based team training has not been studied for TBC, however it has been useful in improving performance in other scenarios (36).

\section{The proceduralist and location}

The proceduralist performing $\mathrm{TBC}$ should be a trained interventional pulmonologist with prior supervised training in performing this procedure (4). An assistant who only manages the bronchial blocker at all times should be present. TBC should be performed in an operating room or a dedicated bronchoscopy suite with equipment to handle emergencies ready and immediately available (4). This equipment includes; an airway cart with multiple sizes of endotracheal tubes, oral airways, direct laryngoscopy equipment, a code cart, equipment to insert chest tubes emergently and to control endobronchial bleeding like balloon blockers of various sizes $(1,7)$.

\section{A proposed procedure-step by step}

(I) A detailed procedural pause/time out should be performed, during which the procedural plan, target lobes, assigned roles, and a backup safety plan for potential complications should also be reviewed.

(II) Abnormal areas seen on pre-procedure computed tomography (CT) scan should be targeted with attempts made to take each biopsy from a different segment. As per standard of care, 3-5 biopsies should be obtained per patient, all from different segments and at least from two different lobes. Only one lung should be biopsied.

(III) A 7 Fr or 9 Fr bronchial blocker should be used for prophylactic airway occlusion to avoid contamination of other airways with blood during the procedure. This blocker should be preferably placed in the 
target lobe from outside the ETT lumen to provide space for ventilation through the ETT as well as for movement of the bronchoscope. As described in previous publications, the ETT can first be threaded over the bronchoscope and secured followed by tightening the snare of the bronchial blocker around the tip of the bronchoscope allowing it to be driven to the target lobe with the bronchoscope and deployed $(2,5)$. The bronchial blocker should be checked for any leaks by inflating air through it under saline before start of the procedure.

(IV) After deploying the bronchial blocker in the desired location, the ETT can be advanced over a flexible bronchoscope (large therapeutic channel scope for passing instruments as well as providing space for continuous suction). A wire reinforced endotracheal tube can be considered as it allows for ease of manually intubating the right or left mainstem in case of severe bleeding during the procedure.

(V) Oxygen is delivered per ETT. Consider keeping the cuff deflated unless indicated to assist ventilation. An anesthesiologist or certified registered nurse anesthetist (CRNA) can provide deep intravenous sedation, and adequate spontaneous respiratory effort should be maintained as much as possible. Paralytics should be used only if adequate sedation and spontaneous assisted ventilation could not be safely co-maintained or if the procedure is performed under rigid bronchoscopy.

(VI) A patient's tolerance for TBC can be checked by a trial of occlusion of the targeted lobe by balloon blocker for 3-4 minutes. If the patient desaturates significantly raising concerns, the procedure can be aborted at that point. This practice has not been studied in the literature; however, it simulates postTBC occlusion of target segment with blood and atelectasis that can cause decompensation in some patients.

(VII) Real-time fluoroscopy should be used in all cases to guide the cryobiopsy probe placement.

(VIII) A carbon dioxide-cooled cryoprobe, 1.9 or $2.4 \mathrm{~mm}$ in diameter should be advanced under fluoroscopic guidance until it meets resistance or the probe is seen to reach the pleura.

(IX) The cryoprobe should be then pulled back $1 \mathrm{~cm}$ and activated for 5 seconds. The cryoprobe and bronchoscope are then pulled out of the airway en bloc. This technique targets the area of the lung parenchyma that is best suited for biopsy as it has less risk of pneumothorax (safer distance from parietal pleura), less chance of bleeding (avoids mediumsized arteries that lack complete cartilage plates) and has higher yield for diagnosis of UIP (proximity to the secondary lobule and subpleural area) $(4,37)$. The prophylactic bronchial blocker should be inflated by the bedside assistant as soon as each cryobiopsy is pulled out along with the bronchoscope.

(X) The cryobiopsy should be submerged in room temperature saline to rapidly thaw and release the tissue, after which the cryoprobe is removed from the bronchoscope. This biopsy should be gently transferred to formalin with the help of a needle.

(XI) The bronchoscope should be quickly reintroduced into the airway to confirm the inflation and position of the bronchial blocker occluding the biopsied airway and blocker should be deflated when appropriate.

(XII) Strategies to achieve hemostasis should be carried out as needed, including iced saline, suction, tamponade and re-inflation of blocker, patient repositioning, etc.

(XIII) After satisfactory hemostasis is obtained it is then safe to proceed with the next cryobiopsy.

(XIV) At the discretion of the operator, the process can be repeated in different locations in the same lobe to achieve up to 3-5 biopsies.

\section{Intra-procedural safety practices}

\section{Prevention of bleeding}

Intra-operative bleeding is the most feared complication of TBC, and balloon blockade is suggested to be prophylactically performed to prevent the same $(4,8,38-41)$. A recent meta-analysis of 27 studies showed that the incidence of combined moderate and severe bleeding was $14.2 \%$ (95\% CI, 7.9-21.9\%) (42). The pooled estimate of TBCB procedure-related moderate/severe bleeding was $39 \%$ in one meta-analysis (43), but another systematic review reported a $12 \%$ probability of moderate bleeding (8). In another meta-analysis of 12 studies including 383 patients, there were no reported episodes of severe bleeding. The likelihood of developing moderate bleeding in this meta-analysis was 0.12 (95\% CI, 0.02-0.25) (8). Analyses of bleeding rates are complicated due to significant heterogeneity across studies, including procedural technique, 
Table 2 Key points: intra-operative safety checklist

Intraoperative safety checklist

1. Anesthesia: deep sedation or general anesthesia with the use of an endotracheal tube (8.5 mm diameter or larger)

2. Proceduralist-with prior supervised training in performing transbronchial cryobiopsy at high volume center

3. Location-operating room or a dedicated bronchoscopy suite

4. Procedural plan: target site planned before the procedure, equipment checked and verified (bronchial blocker, cryoprobe)

5. Staff preparation-roles assigned beforehand to improve communication

6. Personnel needed: Bronchoscopist, an assistant to the bronchoscopist who operates the bronchial blocker, bedside scrub technician/nurse/respiratory therapist to assist with managing the cryoprobe, retrieving cryobiopsies after they are thawed and assist the bronchoscopist in supplies to maintain hemostasis

7. Safety equipment in the room: an airway cart with multiple sizes of endotracheal tubes, oral airways, direct laryngoscopy equipment; a code cart, chest tubes insertion kit; cold saline, balloon blockers of various sizes

post-biopsy monitoring, and inconsistent definitions of the severity of airway bleeding (43). A commonly used definition originated in 2006 by Ernst et al. describing airway bleeding as no bleeding, mild, moderate and severe bleeding (20). Since 2016, there have been cases reported of massive hemoptysis and life-threatening bleeding $(6,11)$. Two reported cases required conversion to rigid bronchoscopy for blood clot extraction in one study and two cases of mortality were reported within 30 days of the procedure in another recent study $(6,40)$. Thus, careful patient selection and correction of bleeding diathesis is paramount. Prophylactic placement of a bronchial blocker and endotracheal intubation for patients is recommended $(4,6)$.

\section{Pneumothorax}

Pneumothorax is a complication associated with TBC with a reported rate from $1 \%$ up to $30 \%$ in the literature (4). In a meta-analysis of 30 studies that included 1443 patients, the pneumothorax rate was $9.4 \%$ (95\% CI, $6.7-12.5 \%)(42)$. The same average rate of $9.5 \%(5.9-14.9 \%)$ was reported on a prior meta-analysis of 13 studies (44). Of all patients developing a pneumothorax, not all of them will require a chest tube for drainage. In one meta-analysis of 15 studies including 994 patients, out of a 100 pneumothoraces (10\%), 70 (70\%) required chest tube drainage (33). The risk of pneumothorax increases with biopsies taken at a close distance to the pleura, UIP histological pattern, and fibrosing DPLD $(28,33)$. Ravaglia et al. identified several factors that increased the risk of pneumothorax: when samples were taken from different sites or the lower lobes, use of a $2.4 \mathrm{~mm}$ probe, and obtaining more than three samples. This risk can be decreased by the use of fluoroscopic guidance during $\mathrm{TBC}$ as well as using a $1.9 \mathrm{~mm}$ probe instead of the $2.4 \mathrm{~mm}$ probe $(10,45)$. Use of $1.9 \mathrm{~mm}$ probe also allows for some use of suction around the cryoprobe as well as less chances of the cryoprobe getting stuck at a distal carina and not reaching the target subpleural area when advanced outside the scope to obtain TBC (4).

Key points to consider while performing TBC are summarized in Table 2.

\section{Post procedural considerations}

The management of patients post-TBC includes the assessment for any procedural complications, especially pneumothorax. Several systematic reviews and meta-analyses of TBC complications have identified a pneumothorax risk of $6.8-20 \%(33,43-45)$. It was as high as $28 \%$ in a small prospective study of patients with fibrotic DPLD (29). Thus, the Cryobiopsy Working Group suggests a post-procedural CXR or ultrasound exam either immediately (if symptomatic) or 2 hours after the end of the procedure (if asymptomatic) (4). A small prospective pilot study found that chest ultrasound is highly sensitive and specific for the diagnosis of pneumothorax after lung biopsy, identified by both the absence of lung sliding and a "stratosphere sign" in M-mode (46).

Patients should be observed in the post-procedure recovery area as per local institutional guidelines, and hospitalization can be considered for individual patients with post-procedural complications. The median time to the first adverse event after TBC is 0.6 days (20); thus, TBC could be performed in an outpatient setting. 
Table 3 Key points: post-procedure safety checklist

Post-procedure checklist

* CXR or ultrasound exam either immediately (if symptomatic) or 2 hours after the end of the procedure (if asymptomatic)

* Observation in the recovery area as per local institutional guidelines

* Monitor for any complications, such as respiratory failure, seizures, atrial fibrillation, hemoptysis, pneumothorax

Median hospitalization length of stay post-TBC is 2.6 days (20) but can increase to a mean of 6 days in patients with post-procedure pneumothorax (3). In a large series of 699 patients who underwent TBC, $19.2 \%$ of patients experienced pneumothorax and $70.1 \%$ of patients with a pneumothorax required chest tube drainage (8). Most patients are monitored for at least $2-3$ hours postprocedure $(4,8)$; it is essential to recognize that post-TBC pneumothorax can be delayed (46). Thus, a follow-up phone call at 24 hours could be considered (47). The concerning signs and symptoms include dyspnea, palpitations, chest pain, tachycardia, hypotension, and hypoxemia. Patients are also instructed to notify their provider if any of these symptoms develop post-discharge.

The 30-day mortality after TBC has been reported but can be variable, $0.4 \%$ per a study of 699 patients by Ravaglia et al. (8), $2.0 \%$ percent per another study of 197 patients by Pannu et al. (6) and $1.9 \%$ per a study of 159 patients by Cooley et al. (7). Deaths post-TBC are commonly due to acute exacerbation of IPF and are significantly higher when TBC is performed on inpatients $(25 \%$ vs. $2.0 \%$ and $5.9 \%$ vs. $1.9 \%)(6,8,28,33)$. This is a similar trend when compared to post SLB mortality. In patients with ILD who underwent SLB, the in-hospital mortality was $1.7 \%$ for elective surgical lung biopsies and significantly higher for nonelective procedures (16\%), based on a 2000-2011 Nationwide Inpatient Sample (48).

A review of 15 TBC studies including 994 patients identified other complications: 4 transient respiratory failures $(0.4 \%), 1$ death $(0.1 \%), 1$ acute exacerbation $(0.1 \%)$, and 1 prolonged air leak (0.1\%) (33). Another review of 11 TBC studies, including 731 patients identified only one patient with acute exacerbation and one procedure-related death (43). Other side effects that occurred in 9 of 699 $(1.3 \%)$ patients in one study included: 4 transient respiratory failure, one empyema, one seizure, one atrial fibrillation, one pneumomediastinum, and one hemoptysis (8). Hospitalized patients with ILD are at higher risk of postTBC pneumothorax, persistent air leak, ICU transfer in addition to higher 30-day mortality (7).
Key points to consider after performing TBC are summarized in Table 3.

\section{Summary and conclusion}

TBC can provide the benefit of increasing diagnostic yield; however, modifiable procedural aspects should be optimized and standardized to decrease the risk of complications. Patient selection, procedural technique, and careful postoperative monitoring are all key to mitigating the risks. Bleeding diathesis, obesity, pulmonary hypertension, and lung function should be carefully evaluated in potential candidates for TBC. The intraprocedural use of fluoroscopy and prophylactic endobronchial balloon blockade are recommended. Postoperative care includes obtaining a CXR or ultrasound exam to evaluate for pneumothorax in addition to monitoring for any other complications. This updated review of safety practices in performing TBC can be applied to decrease potential adverse outcomes.

\section{Acknowledgments}

Funding: None.

\section{Footnote}

Provenance and Peer Review: This article was commissioned by the Guest Editors (Kyle Hogarth \& Jonathan Kurman) and the editorial office for the series "Interventional pulmonology and advanced bronchoscopy" published in Shanghai Chest. This article has undergone external peer review.

Conflicts of Interest: All authors have completed the ICMJE uniform disclosure form (available at http://dx.doi. org/10.21037/shc.2020.03.05). The series "Interventional pulmonology and advanced bronchoscopy" was commissioned by the editorial office without any funding or sponsorship. The authors have no other conflicts of interest to declare. 
Ethical Statement: The authors are accountable for all aspects of the work in ensuring that questions related to the accuracy or integrity of any part of the work are appropriately investigated and resolved.

Open Access Statement: This is an Open Access article distributed in accordance with the Creative Commons Attribution-NonCommercial-NoDerivs 4.0 International License (CC BY-NC-ND 4.0), which permits the noncommercial replication and distribution of the article with the strict proviso that no changes or edits are made and the original work is properly cited (including links to both the formal publication through the relevant DOI and the license). See: https://creativecommons.org/licenses/by-nc-nd/4.0/.

\section{References}

1. Babiak A, Hetzel J, Krishna G, et al. Transbronchial cryobiopsy: a new tool for lung biopsies. Respiration 2009;78:203-8.

2. Lentz RJ, Argento AC, Colby TV, et al. Transbronchial cryobiopsy for diffuse parenchymal lung disease: a state-ofthe-art review of procedural techniques, current evidence, and future challenges. J Thorac Dis 2017;9:2186-203.

3. Tomassetti S, Wells AU, Costabel U, et al. Bronchoscopic lung cryobiopsy increases diagnostic confidence in the multidisciplinary diagnosis of idiopathic pulmonary fibrosis. Am J Respir Crit Care Med 2016;193:745-52.

4. Hetzel J, Maldonado F, Ravaglia C, et al. Transbronchial cryobiopsies for the diagnosis of diffuse parenchymal lung diseases: expert statement from the cryobiopsy working group on safety and utility and a call for standardization of the procedure. Respiration 2018;95:188-200.

5. Lentz RJ, Taylor TM, Kropski JA, et al. Utility of flexible bronchoscopic cryobiopsy for diagnosis of diffuse parenchymal lung diseases. J Bronchology Interv Pulmonol 2018;25:88-96.

6. Pannu J, Roller LJ, Maldonado F, et al. Transbronchial Cryobiopsy for Diffuse Parenchymal Lung Disease: 30and 90-Day Mortality. Eur Respir J 2019;54:1900337.

7. Cooley J, Balestra R, Aragaki-Nakahodo AA, et al. Safety of performing transbronchial lung cryobiopsy on hospitalized patients with interstitial lung disease. Respir Med 2018;140:71-6.

8. Ravaglia C, Wells AU, Tomassetti S, et al. Diagnostic yield and risk/benefit analysis of trans-bronchial lung cryobiopsy in diffuse parenchymal lung diseases: a large cohort of 699 patients. BMC Pulm Med 2019;19:16.
9. Berim IG, Saeed AI, Awab A, et al. Radial probe ultrasound-guided cryobiopsy. J Bronchology Interv Pulmonol 2017;24:170-3.

10. Maldonado F, Danoff SK, Wells AU, et al. Transbronchial Cryobiopsy for the Diagnosis of Interstitial Lung Diseases: CHEST Guideline and Expert Panel Report. Chest 2020;157:1030-42.

11. DiBardino DM, Haas AR, Lanfranco AR, et al. High complication rate after introduction of transbronchial cryobiopsy into clinical practice at an academic medical center. Ann Am Thorac Soc 2017;14:851-7.

12. Pannu JK, Rickman OB, Lentz RJ, et al. Persistent focal pulmonary opacity elucidated by transbronchial cryobiopsy: a case for larger biopsies. Respirol Case Rep 2019;7:e00410.

13. Raghu G, Remy-Jardin M, Myers JL, et al. Diagnosis of idiopathic pulmonary fibrosis. An official ATS/ERS/JRS/ ALAT clinical practice guideline. Am J Respir Crit Care Med 2018;198:e44-e68.

14. Schuhmann M, Bostanci K, Bugalho A, et al. Endobronchial ultrasound-guided cryobiopsies in peripheral pulmonary lesions: a feasibility study. Eur Respir J 2014;43:233-9.

15. Fruchter O, Fridel L, Rosengarten D, et al. Transbronchial cryo-biopsy in lung transplantation patients: first report. Respirology 2013;18:669-73.

16. Lentz RJ, Fessel JP, Johnson JE, et al. Transbronchial cryobiopsy can diagnose constrictive bronchiolitis in veterans of recent conflicts in the Middle East. Am J Respir Crit Care Med 2016;193:806-8.

17. Fruchter O, Fridel L, Rosengarten D, et al. Transbronchial cryobiopsy in immunocompromised patients with pulmonary infiltrates: a pilot study. Lung 2013;191:619-24.

18. Ryu JH, Daniels CE, Hartman TE, et al., editors. Diagnosis of interstitial lung diseases. Mayo Clinic Proceedings; 2007: Elsevier.

19. Wells A, Hirani N. Interstitial lung disease guideline. Thorax 2008;63:v1-v58.

20. Ernst A, Eberhardt R, Wahidi M, et al. Effect of routine clopidogrel use on bleeding complications after transbronchial biopsy in humans. Chest 2006;129:734-7.

21. Papin TA, Lynch III JP, Weg JG. Transbronchial biopsy in the thrombocytopenic patient. Chest 1985;88:549-52.

22. Herth FJ, Becker H, Ernst A. Aspirin does not increase bleeding complications after transbronchial biopsy. Chest 2002;122:1461-4.

23. Pathak V, Allender JE, Grant MW. Management of anticoagulant and antiplatelet therapy in patients 
undergoing interventional pulmonary procedures. Eur Respir Rev 2017;26:170020.

24. Du Rand I, Blaikley J, Booton R, et al. British Thoracic Society guideline for diagnostic flexible bronchoscopy in adults: accredited by NICE. Thorax 2013;68:i1-i44.

25. Chan J, Yeung Y, Sin K, et al. Guidelines of procedural and sedation safety in flexible bronchoscopy and pleuroscopy. Hong Kong Thoracic Society, American College of Chest Physicians (Hong Kong and Macau Chapter), Hong Kong Lung Foundation 2016.

26. Kim JH, Baek CH, Min JY, et al. Desmopressin improves platelet function in uremic patients taking antiplatelet agents who require emergent invasive procedures. Ann Hematol 2015;94:1457-61.

27. Bernasconi M, Koegelenberg CF, Koutsokera A, et al. Iatrogenic bleeding during flexible bronchoscopy: risk factors, prophylactic measures and management. ERJ Open Res 2017;3:00084-2016.

28. Casoni GL, Tomassetti S, Cavazza A, et al. Transbronchial lung cryobiopsy in the diagnosis of fibrotic interstitial lung diseases. PLoS One 2014;9:e86716.

29. Hagmeyer L, Theegarten D, Treml M, et al. Validation of transbronchial cryobiopsy in interstitial lung diseaseinterim analysis of a prospective trial and critical review of the literature. Sarcoidosis Vasc Diffuse Lung Dis 2016;33:2-9.

30. Kropski JA, Pritchett JM, Mason WR, et al. Bronchoscopic cryobiopsy for the diagnosis of diffuse parenchymal lung disease. PLoS One 2013;8:e78674.

31. Galiè N, Humbert M, Vachiery JL, et al. 2015 ESC/ERS guidelines for the diagnosis and treatment of pulmonary hypertension: the Joint Task Force for the Diagnosis and Treatment of Pulmonary Hypertension of the European Society of Cardiology (ESC) and the European Respiratory Society (ERS): endorsed by: Association for European Paediatric and Congenital Cardiology (AEPC), International Society for Heart and Lung Transplantation (ISHLT). Eur Heart J 2016;37:67-119.

32. Devaraj A, Wells AU, Meister MG, et al. Detection of pulmonary hypertension with multidetector CT and echocardiography alone and in combination. Radiology 2010;254:609-16.

33. Ravaglia C, Bonifazi M, Wells AU, et al. Safety and diagnostic yield of transbronchial lung cryobiopsy in diffuse parenchymal lung diseases: a comparative study versus video-assisted thoracoscopic lung biopsy and a systematic review of the literature. Respiration 2016;91:215-27.
34. Cortadellas MC, Oezkan F, Boerner EB, et al. Diagnosing Interstitial lung dieseases via cryobiopsy in rigid bronchoscopy: Preliminary results. Eur Respir J 2016 48:PA4668.

35. Risser DT, Rice MM, Salisbury ML, et al. The potential for improved teamwork to reduce medical errors in the emergency department. Ann Emerg Med 1999;34:373-83.

36. Hunt EA, Shilkofski NA, Stavroudis TA, et al. Simulation: translation to improved team performance. Anesthesiology clinics 2007;25:301-19.

37. Colby TV, Tomassetti S, Cavazza A, et al. Transbronchial cryobiopsy in diffuse lung disease: update for the pathologist. Arch Pathol Lab Med 2017;141:891-900.

38. Poletti V, Hetzel J. Transbronchial cryobiopsy in diffuse parenchymal lung disease: need for procedural standardization. Respiration 2015;90:275-8.

39. Pajares V, Puzo C, Castillo D, et al. Diagnostic yield of transbronchial cryobiopsy in interstitial lung disease: a randomized trial. Respirology 2014;19:900-6.

40. Hagmeyer L, Theegarten D, Wohlschläger J, et al. The role of transbronchial cryobiopsy and surgical lung biopsy in the diagnostic algorithm of interstitial lung disease. Clin Respir J 2016;10:589-95.

41. Sharp C, McCabe M, Adamali H, et al. Use of transbronchial cryobiopsy in the diagnosis of interstitial lung disease-a systematic review and cost analysis. QJM 2017;110:207-14.

42. Sethi J, Ali MS, Mohananey D, et al. Are Transbronchial Cryobiopsies Ready for Prime Time?: A Systematic

Review and Meta-Analysis. J Bronchology Interv Pulmonol 2019;26:22-32.

43. Johannson KA, Marcoux VS, Ronksley PE, et al. Diagnostic yield and complications of transbronchial lung cryobiopsy for interstitial lung disease. A systematic review and metaanalysis. Ann Am Thorac Soc 2016;13:1828-38.

44. Iftikhar IH, Alghothani L, Sardi A, et al. Transbronchial lung cryobiopsy and video-assisted thoracoscopic lung biopsy in the diagnosis of diffuse parenchymal lung disease. A meta-analysis of diagnostic test accuracy. Ann Am Thorac Soc 2017;14:1197-211.

45. Dhooria S, Sehgal IS, Aggarwal AN, et al. Diagnostic yield and safety of cryoprobe transbronchial lung biopsy in diffuse parenchymal lung diseases: systematic review and meta-analysis. Respir Care 2016;61:700-12.

46. Viglietta L, Inchingolo R, Pavano C, et al. Ultrasonography for the diagnosis of pneumothorax after transbronchial lung cryobiopsy in diffuse parenchymal lung diseases. Respiration 2017;94:232-6. 
47. Bango-Álvarez A, Ariza-Prota M, Torres-Rivas H, et al. Transbronchial cryobiopsy in interstitial lung disease: experience in 106 cases-how to do it. ERJ Open Res 2017;3:00148-2016.

doi: 10.21037/shc.2020.03.05

Cite this article as: Pannu J, Holden V, Ghattas C. Transbronchial cryobiopsy: how to do it safely? Shanghai Chest 2021;5:16.
48. Hutchinson JP, Fogarty AW, McKeever TM, et al. Inhospital mortality after surgical lung biopsy for interstitial lung disease in the United States. 2000 to 2011. Am J Respir Crit Care Med 2016;193:1161-7. 